\title{
Costos de atención médica de las enfermedades atribuibles al consumo de tabaco en América: revisión de la literatura
}

\author{
Luz Myriam Reynales-Shigematsu, Dr en C.(I)
}

\begin{abstract}
Reynales-Shigematsu LM.
Costos de atención médica de las enfermedades atribuibles al consumo de tabaco en las Américas: revisión de la literatura. Salud Publica Mex 2006;48 supl I:S190-S200.

\section{Resumen}

Con el objetivo de recopilar la información de los estudios de costos de atención médica atribuibles al consumo de tabaco publicados en la literatura científica y de evaluar las diferentes estrategias metodológicas utilizadas en la estimación, se revisaron los trabajos identificados a través de MedLINE y de las referencias bibliográficas de los libros publicados por el Banco Mundial, la Organización Mundial de la Salud, la Organización Panamericana de la Salud, el Grupo de la Investigación Interdisciplinaria de la Salud de Canadá, así como el documento técnico utilizado en el litigio enfrentado por el estado de Minnesota, Estados Unidos de América, contra la industria tabacalera. Se incluyeron todos los estudios publicados en la literatura sobre el tema desde hace más de 25 años. Se obtuvo información de la población de estudio, la perspectiva de costeo, el tipo de análisis para la estimación de los costos de atención médica y la metodología de atribución de costos al consumo de tabaco. Además, se agregó un comentario de los hallazgos relevantes y de las limitaciones de cada uno de los estudios. Los costos anuales de atención médica atribuibles al consumo de tabaco oscilan entre 6 a $14 \%$ de los gastos personales en salud. Desde la primera publicación hasta la fecha se observa un progreso en la metodología de la estimación, no sólo desde la perspectiva epidemiológica -que afina la atribución de los costos al factor de riesgo- sino desde la económica -que amplía la estimación de los cos-
\end{abstract}

Reynales-Shigematsu LM.

Literature review of health care costs of diseases attributable to tobacco consumption in the Americas. Salud Publica Mex 2006;48 suppl I:S190-S200.

\begin{abstract}
The objective of this study was to compile information from published scientific literature about health care costs attributable to tobacco consumption and evaluate the different methodological strategies used in calculating estimations. Sources included MedLINE, bibliographical references from books published by the World Bank, the World Health Organization, the Panamerican Health Organization, the Interdisciplinary Health Research Group of Canada, as well as technical documenation used by the state of Minnesota, United States of America, in litigation against the tobacco industry. All of the studies published about this issue over the last 25 years or more were included. Information was obtained with respect to the study population, the cost perspective, the type of analysis used for estimating health care costs and methodology for attributing costs to tobacco consumption. In addition, comments with regard to the relevant findings and the limitations of each of the studies were added.Annual health care costs attributable to tobacco use vary between 6 and $14 \%$ of personal health expenses. In the period between the first publication and today, progress has been seen in the methodology used for calculating estimations, not only from the epidemiological perspective which improves the accuracy of the attribution of costs to risk factors, but from the economic perspective which broadens the estimation of costs from a social perspective. It is concluded that tobacco consumption leads to
\end{abstract}

Este proyecto se llevó a cabo con financiamiento del Consejo Nacional de Ciencia y Tecnología (SEP-CONACyT 2002-I No. 40593), el Institute for Global Tobacco Control (IGTC) de Johns Hopkins Bloomberg School of Public Health (Fogarty Center ROI-HL-73699) y el Instituto Mexicano del Seguro Social (FOFOI-IMSS 2002-322-003I).

(I) Departamento de Investigación sobre Tabaco. Centro de Investigación en Salud Poblacional, Instituto Nacional de Salud Pública. Cuernavaca, Morelos, México.

Fecha de aprobado: 17 de abril de 2006

Solicitud de sobretiros: Luz Myriam Reynales-Shigematsu. Instituto Nacional de Salud Pública. Av. Universidad 655 , Col. Santa María Ahuacatitlán, 62508 Cuernavaca, Morelos. México.

Correo electrónico: Ireynales@correo.insp.mx 
tos desde una perspectiva social. Se concluye que el consumo de tabaco deriva en altos costos de atención médica, y representa un alto costo social por las muertes prematuras que genera en la sociedad.

Palabras clave: costos de atención médica; tabaco; economía de la salud high health care costs, involves a cost to employers due to productivity losses and worker disability, and represents a high social cost resulting from the occurrence of premature deaths in the society.

Key words: health care costs; tobacco; health economy
E 1 tabaco mata hoy a 1 de cada 10 adultos en el mundo. Para el año 2030, la proporción ascenderá a 1 de cada 6, lo que equivale a 10 millones de defunciones anuales, cifra superior a la de mortalidad por cualquier otra causa. ${ }^{1}$ Se estima que en el mundo existen 1100 millones de fumadores, 300 millones en los países desarrollados y 800 en los países en desarrollo. ${ }^{1}$

La prevalencia de tabaquismo en la población del continente americano, entre 1996 y 1999, osciló entre 22 y 40\%; en algunas zonas urbanas más de la mitad de los jóvenes fuman. La distribución porcentual entre los hombres fumadores oscila entre 26 y $47 \%$, y en las mujeres entre 16 y $36 \% .{ }^{2,3}$ Es importante no perder de vista que la epidemia de tabaquismo se ha desplazado a los países de medianos y bajos ingresos, los cuales concentran en la actualidad a $80 \%$ de la población fumadora en el mundo. ${ }^{1}$ Los efectos en la morbilidad, la mortalidad y el bienestar de la población no son evidentes todavía, de ahí la tendencia de los gobiernos a subestimar los costos atribuibles al consumo de tabaco y a sobreestimar la importancia fiscal del tabaco en la economía nacional. ${ }^{1,2}$

Las consecuencias asociadas con el consumo de tabaco van más allá de los daños a la salud individual y colectiva. La carga económica que su consumo impone a la sociedad involucra no sólo los costos de atención médica de las enfermedades que causa, sino los días de incapacidad, los casos de invalidez y los años de vida potencial perdidos por muerte prematura. ${ }^{4,5,6}$ Desde hace décadas se conoce que el consumo de tabaco produce enfermedad y muerte prematura a causa de más de 25 enfermedades, entre ellas diferentes tipos de cáncer, enfermedades respiratorias y cardiovasculares, ${ }^{7-11}$ y otras de ocurrencia más frecuente como neumonías, cataratas y periodontitis. ${ }^{12}$

El impacto económico del consumo de tabaco en las sociedades, incluyendo los gastos de atención médica y las pérdidas de productividad, se ha documentado en la literatura desde hace más de 25 años. Por lo tanto, el propósito de este trabajo es recopilar la infor- mación de los estudios de costos de atención médica atribuibles al consumo de tabaco publicados en la literatura científica, evaluar las diferentes estrategias metodológicas utilizadas en la estimación y presentar los principales resultados y conclusiones de los autores.

\section{Fuentes de información}

Se revisaron los estudios identificados a través de MedLINE y de las referencias bibliográficas de los libros publicados por el Banco Mundial (BM), la Organización Mundial de la Salud (OMS), la Organización Panamericana de la Salud (OPS) y el Grupo de la Investigación Interdisciplinaria de la Salud de Canadá (GRIS), así como el documento técnico utilizado en el litigio enfrentado por el estado de Minnesota, en Estados Unidos de América (EUA), contra la industria tabacalera.

\section{Metodología para la obtención de los datos}

Se obtuvo información sobre la población de estudio, la perspectiva de costeo, el tipo de análisis para la estimación de los costos de atención médica y la metodología de atribución de costos al consumo de tabaco. Además, se agregó un comentario de los hallazgos relevantes y de las limitaciones de cada uno de los estudios.

\section{Revisión}

La estimación de los costos brutos en salud atribuibles al tabaco (que corresponde a los gastos asociados con el tratamiento de las enfermedades atribuibles al consumo de tabaco) para los países desarrollados se encuentra entre 0.10 a $1.1 \%$ del producto interno bruto (PIB); en los países de bajos y medianos ingresos los datos son muy limitados pero algunos resultados sugieren que podrían ser mayores que los de países desarrollados. ${ }^{13}$ 


\section{Estudios transversales}

Costos atribuibles al consumo de tabaco en

Estados Unidos de América y Canadá

El cuadro I resume las estimaciones de costos anuales atribuibles al consumo de tabaco realizadas en EUA y Canadá utilizando diversas metodologías para la atribución de tales costos.

Hendrick $^{14}$ fue uno de los pioneros de los estudios de costos relacionados con el consumo de tabaco en Canadá. Estimó los costos de atención médica de cuatro enfermedades y utilizó las tasas de mortalidad de cada una en EUA para estimar los costos atribuibles al consumo de tabaco. Posteriormente los extrapoló a EUA utilizando la razón del PIB para estos dos países (cuadro I).

Kristein ${ }^{15}$ encontró que los fumadores intensos (aquellos que consumen más de 20 cigarrillos al día) se hospitalizan 1.5 veces más que los no fumadores.

Luce y Schweitzer ${ }^{16}$ incluyeron en su estimación de costos a enfermedades como el cáncer, las cardiovasculares y respiratorias, y los accidentes. Por primera vez introdujeron el concepto epidemiológico de fracción atribuible (se refiere al porcentaje de la enfer- medad que podría reducirse si el factor causal [tabaco] fuese eliminado) en la estimación de costos de atención médica atribuibles al consumo de tabaco.

La Oficina de Investigación y tecnología, de EUA (OTA, por sus siglas en inglés) ${ }_{1}^{17}$ estimó los costos atribuibles al tabaco teniendo en cuenta únicamente los cánceres y las enfermedades cardiovasculares y respiratorias. Para ello utilizó las tasas de mortalidad relacionadas con el consumo de tabaco según género y grupo de edad, proporcionadas por la Sociedad Americana del Cáncer (ACS) y por el Centro Nacional de Estadísticas de EUA (NCHC) aplicándolas a estimaciones de costos previamente realizadas por Kopstein y Hodgson.

Rice y colaboradores ${ }^{18}$ introdujeron una nueva metodología para atribuir los costos al tabaco basada en el riesgo atribuible de utilización de servicios médicos (hospitalización y atención ambulatoria) de los fumadores actuales, y de los no fumadores que tenían enfermedades cardiovasculares, respiratorias y cáncer. Ellos aplicaron la proporción de la utilización, de acuerdo con el consumo de tabaco, a los costos previamente estimados por Kopstein y Hogdson. Estas razones fueron estimadas a partir de la Encuesta Nacional de Salud de EUA (NHIS). El algoritmo utilizado en este

Cuadro I

Estimaciones anuales de los costos en SALUd atribuibles al consumo de tabaco. Estados Unidos Y CANADÁ

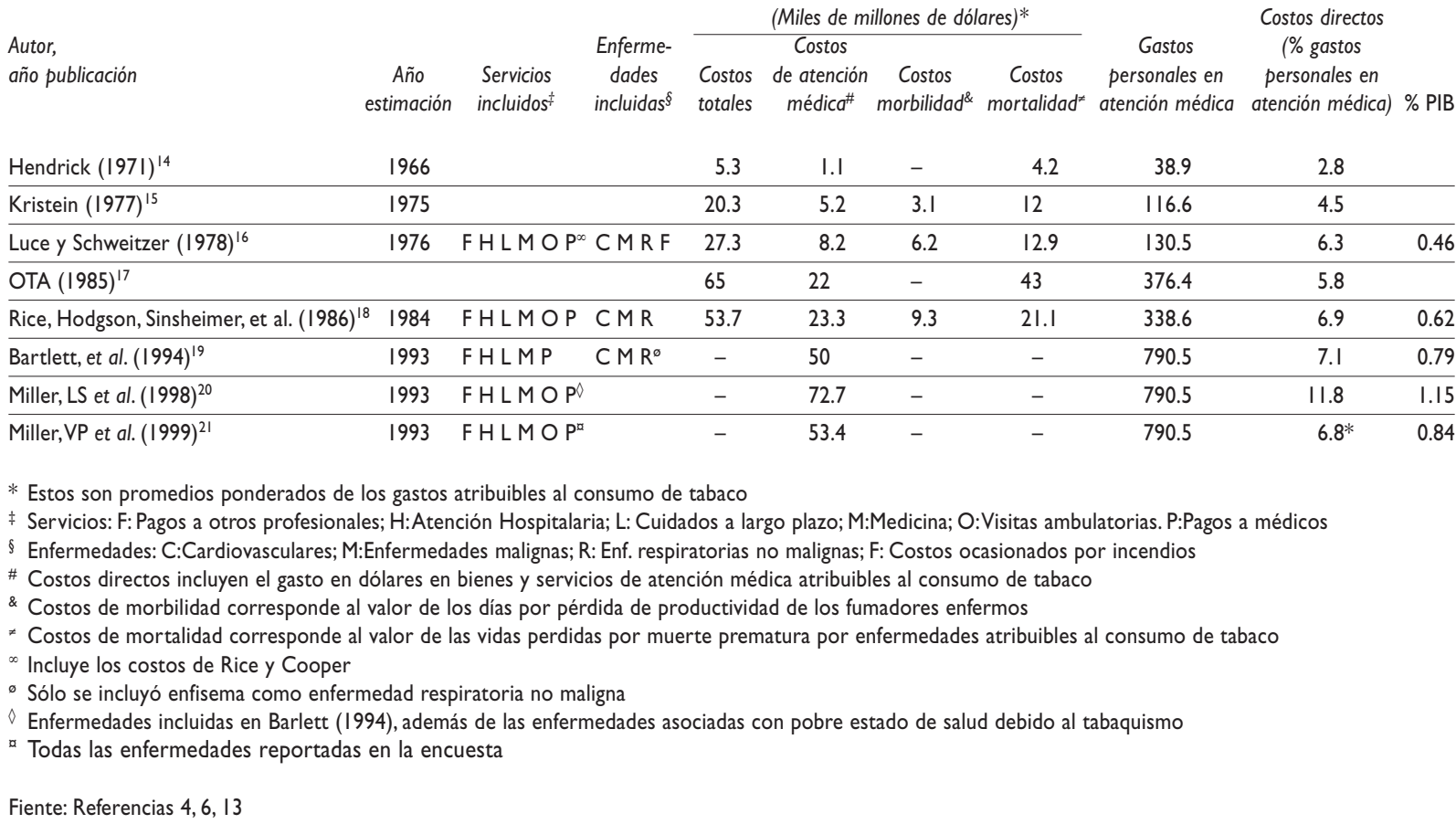


estudio fue la base metodológica para desarrollar el Programa Morbilidad, Mortalidad y Costos Atribuibles al Consumo de Tabaco (SAMMEC). Con éste se lograron estimar los costos para casi 50 estados de EUA; sin embargo, la literatura refiere que estos costos se encuentran subestimados debido a que los riesgos relativos de los días de hospitalización y visitas médicas fueron obtenidos a partir de la Encuesta Nacional de Salud, donde sólo discrimina a los fumadores actuales $\mathrm{y}$ a los no fumadores y no tiene en cuenta a los ex fumadores, quienes tienen más riesgo de presentar las efermedades. Esta es la razón por la cual las estimaciones de los costos directos proporcionadas por el SAMMEC corresponden tan sólo al 3 a 4\% de los gastos personales en salud.

Durante la última década, los investigadores han llamado la atención sobre el desarrollo de modelos econométricos para estimar los costos atribuibles al consumo de tabaco. Estos modelos introducen en detalle el antecedente de tabaquismo y otros factores de riesgo individual que pudieran ser confusores de la estimación de costos.

Barlett y colaboradores ${ }^{19}$ incluyeron en su estimación de costos la enfermedad cardiaca, el enfisema, la arteriosclerosis, las enfermedades cerebro vasculares y los cánceres, así como el efecto en el estado general de salud. En 1994, desarrollaron por primera vez un modelo econométrico de multiecuación para estimar las fracciones atribuibles al tabaquismo (SAF), controlando por consumo de tabaco, condiciones médicas, estado de salud y otras características socioeconómicas. Estas fracciones fueron aplicadas a las estimaciones de costos en salud personal realizadas por la Administración Financiera de los Servicios de Salud (HCFA) teniendo en cuenta la Encuesta Nacional de Gasto en Salud de los Hogares y Proveedores de Salud (NMES).

Miller LS y colaboradores ${ }^{20}$ con los investigadores que habían participado en el estudio de Barlett, mediante un modelo probabilístico probit estimaron las SAF, controlando por características sociodemográficas y de comportamiento de los individuos. De igual manera, se utilizó como fuente de información la NMES para estimar la SAF a escala nacional y el Sistema de Vigilancia Epidemiológica de Factores de Riesgo (BRFSS) para la SAF estatal. Las SAF fueron aplicadas a los gastos médicos estatales de salud y luego fueron sumados para tener la estimación nacional. Miller VP y colaboradores ${ }^{21}$ incluyeron en su estudio todas las categorías de enfermedad. Desarrollaron un modelo econométrico de dos ecuaciones para estimar la fracción de gastos médicos atribuible al tabaquismo ajustada por características sociodemográficas y de comportamiento de los individuos. Estas SAF se aplicaron a los gastos médicos estatales y luego se sumaron para obtener el dato nacional. Se utilizó la NMES para estimar las SAF nacionales y la información del suplemento del uso de tabaco (TUS) del BRFSS. Los gastos estatales se obtuvieron a partir de la HCFA.

Las estimaciones de este estudio son controvertibles en dos puntos relevantes. El primero se refiere al modelo de utilización, donde las fracciones de utilización hospitalaria atribuibles al consumo de tabaco son casi dos veces más que las fracciones de utilización ambulatoria, mientras que en el modelo de costos sucede lo contrario. Los autores explican que esta diferencia implica que los fumadores gastan en promedio más que los no fumadores en visitas ambulatorias pero gastan menos en los días de hospitalización. Esta explicación no es del todo convincente, ya que implicaría que los costos de las estancias hospitalarias relacionados con el consumo de tabaco son menos prolongadas que otras estancias. Esto significaría que las enfermedades atribuibles al consumo de tabaco utilizan menos recursos hospitalarios que otras, y esta situación solamente podría suceder si los fumadores (y sus médicos) utilizan menos recursos en las hospitalizaciones, o porque los fumadores se enferman menos o porque simplemente utilizan menos los servicios médicos, en comparación con los no fumadores.

Warner y colaboradores ${ }^{4}$ reanalizan los datos del estudio de Miller VP y colaboradores ${ }^{21}$ para probar las tres hipótesis planteadas anteriormente. Ellos comparan los costos promedio del día de hospitalización de un paciente con cáncer de pulmón y enfermedad obstructiva crónica y uno con enfermedad general. En todos los análisis los costos de las enfermedades atribuibles al consumo de tabaco exceden los costos por enfermedad general. En segundo lugar, no existe diferencia en los costos promedio de hospitalización entre los fumadores y los no fumadores estimados a partir de la NMES. Con estos hallazgos, no habría razón para encontrar que las SAF de utilización sean mayores que las de costos. También refieren que es importante interpretar los resultados al tratar de extrapolarlos a otros países, pues estas estimaciones dependen de las características de la epidemia de tabaquismo, de las diferencias en la eficacia y costo del tratamiento y de las características del sistema de salud.

Zeger y colaboradore ${ }^{23}$ realizaron una estimación de costos atribuibles al consumo de tabaco que fue utilizada como evidencia científica en el litigio presentado por el estado de Minnesota, EUA, en contra de la industria tabacalera en el año 1994. Mediante un modelo estadístico que tiene en cuenta los gastos en el tratamiento de las enfermedades mayores relacionadas con el consumo de tabaco, los gastos por servicios 
médicos relacionados con un deterioro del estado de salud y los gastos en que incurren los hogares, los autores estimaron los costos totales de atención médica. Posteriormente, estimaron la fracción de los costos que razonablemente se atribuyen al factor de riesgo. En términos muy simples, los costos totales fueron reducidos progresivamente por tres factores: a) la prevalencia de tabaquismo en los casos de enfermedad, porque solamente una fracción de la población con una condición médica dada es fumadora; b) la fracción atribuible de expuestos al tabaco, porque aun entre los fumadores, solamente una fracción de su enfermedad es causada por el tabaquismo, y c) la fracción de costos médicos atribuible al consumo de tabaco, porque aun entre los fumadores en los cuales la enfermedad es causada por el tabaquismo, solamente una fracción de sus costos médicos es utilizada para el tratamiento de la enfermedad atribuible al consumo de tabaco (cuadro II).

Johnson y colaboradores ${ }^{24}$ estimaron los costos de atención médica atribuibles al consumo de tabaco te- niendo en cuenta el grupo de enfermedades cardiovasculares (enfermedades del corazón y cerebrovasculares) y respiratorias (cáncer de laringe, de pulmón y enfermedad pulmonar obstructiva crónica), de acuerdo con los datos de la NMES. A partir de un modelo aditivo generalizado se obtuvo la probabilidad de enfermedad. Para la estimación de costos se utilizó un modelo semiparamétrico de dos etapas que permitió estimar la diferencia promedio de costos de atención médica entre las personas que tenían y no tenían la enfermedad. ${ }^{24}$

Dominicci y colaboradore ${ }^{25}$ presentan una metodología novedosa al tratar de estimar la diferencia en los costos promedio de atención médica entre dos poblaciones, con y sin enfermedad, como una función de covariables. Al utilizar la técnica de smooth quantile ratio estimation (SQUARE) estiman la diferencia entre los gastos de atención médica entre los pacientes que sufren enfermedades relacionadas con el consumo de tabaco y los que no. Las estimaciones refieren diferencias entre fumadores que oscilan entre USD $\$ 3000$ a USD $\$ 9000$, comparados con los no fumadores.

Cuadro II

Gastos atribuibles al consumo de tabaco en millones de \$uSd por tipo de enfermedad, edad, SeXo, DE ACUERDO CON LA METODOLOGÍA DE LAS TRES REDUCCIONES

\begin{tabular}{|c|c|c|c|c|c|c|c|c|}
\hline Enfermedad & Sexo & Edad & $\begin{array}{c}\text { Total } \\
\text { \$USD* }\end{array}$ & $\begin{array}{l}\text { ¿Cuántos } \\
\text { fumadores? } \\
\text { (Prevalencia) \% }\end{array}$ & $\begin{array}{l}\text { ¿Cuánto exceso de } \\
\text { enfermedad? (Fracción } \\
\text { atribuible expuestos) \% }\end{array}$ & $\begin{array}{l}\text { ¿Cuántos \$USD } \\
\text { más? (Fracción de } \\
\text { costos atribuible) \% }\end{array}$ & $\begin{array}{l}\text { \$USD atribuibles } \\
\text { al consumo } \\
\text { de tabaco* }\end{array}$ & $\begin{array}{l}\text { Porcentaje } \\
\quad \text { \$USD } \\
\text { atribuibles \% }\end{array}$ \\
\hline \multirow[t]{5}{*}{ Cáncer de Pulmón/ EPOC } & Mujeres & $35-64$ & 101.87 & 0.853 & 0.835 & 0.8 & 58.05 & 56.98 \\
\hline & & $65+$ & 78.7 & 0.938 & 0.963 & 0.72 & 51.04 & 64.86 \\
\hline & Hombres & $35-64$ & 76.35 & 0.875 & 0.683 & 0.77 & 34.91 & 45.72 \\
\hline & & $65+$ & 54.27 & $0.97 \mid$ & 0.659 & 0.32 & 59.00 & \\
\hline & Total & & 311.19 & 0.90 & 0.849 & 0.74 & 175.96 & 56.54 \\
\hline
\end{tabular}

\begin{tabular}{|c|c|c|c|c|c|c|c|c|}
\hline IAM/ECV/Otros & Mujeres & $35-64$ & 252.36 & 0.770 & 0.490 & 0.776 & 73.89 & 29.28 \\
\hline & & $65+$ & 366.49 & 0.426 & 0.097 & 0.533 & 8.07 & 2.20 \\
\hline & Hombres & $35-64$ & 216.99 & 0.957 & 0.608 & 0.762 & 96.21 & 44.34 \\
\hline & & $65+$ & 143.28 & 0.788 & 0.116 & 0.559 & 7.32 & 5.11 \\
\hline & Total & & 979.12 & 0.686 & 0.372 & 0.743 & 185.65 & 18.96 \\
\hline
\end{tabular}

\begin{tabular}{|c|c|c|c|c|c|c|c|c|}
\hline Total & Mujeres & $35-64$ & 354.23 & 0.794 & 0.596 & 0.786 & 131.76 & 37.20 \\
\hline & & $65+$ & 445.19 & 0.517 & 0.375 & 0.685 & 59.12 & 13.28 \\
\hline & Hombres & $35-64$ & 293.34 & 0.936 & 0.626 & 0.763 & 131.14 & 44.71 \\
\hline & & $65+$ & 197.55 & 0.838 & 0.373 & 0.637 & 39.33 & 19.91 \\
\hline & Total & & $|290.3|$ & 0.737 & 0.513 & $0.74 I$ & 361.49 & 28.02 \\
\hline
\end{tabular}

* Millones de USD

EPOC: enfermedad pulmonar obstructiva crónica

IAM: infarto agudo del miocardio

ECV: enfermedad cerebrovascular

Fuente: referencia 23 


\section{Costos atribuibles al consumo de tabaco en Latinoamérica}

El cuadro III resume los estudios hechos en los países de América Latina; éstos muestran que los costos oscilan entre 0.3 y $0.43 \%$ del PIB de cada país. ${ }^{13}$

La Organización Panamericana de la Salud* analizó el impacto económico del tabaquismo en Venezuela, y estimó los costos de atención médica atribuibles al consumo de tabaco, para 1995, en alrededor de $0.3 \%$ del PIB, equivalente a US\$1.436 millones. En términos del porcentaje del gasto en salud, se asume que Venezuela destinó el 20\% para la atención de las enfermedades relacionadas con el consumo de tabaco (cuadro III).

En México, al igual que otros países de Latinoamérica, existe poca información sobre los recursos financieros y materiales utilizados para el diagnóstico y tratamiento de los principales padecimientos crónicos relacionados con el consumo de tabaco, menos aún sobre su impacto económico en el sector salud.

Arredondo y colaboradores ${ }^{26}$ realizaron un estudio en los hospitales de referencia de segundo y tercer nivel de atención de la Secretaría de Salud (SSA) en México, teniendo en cuenta las acciones de manejo diagnóstico y terapéutico referidas por un grupo de expertos y validadas con datos de expedientes clínicos acerca de un caso promedio de enfermedad sin complicaciones. Los autores estiman, desde la perspectiva del proveedor, un costo promedio anual de atención médica por caso de USD\$1 464 para enfermedades cardiovasculares,

\footnotetext{
* OPS. Economic assessment of public policies for tobacco control in Venezuela. Documento Técnico.
}

USD \$628 para cáncer de pulmón y USD $\$ 210$ para enfermedad pulmonar obstructiva crónica (EPOC). Se considera que podrían estar subestimados probablemente debido a la metodología de costos unitarios utilizada. El estudio no hace referencia a la metodología de atribución de costos al consumo de tabaco.

Reynales Shigematsu y colaboradores, ${ }^{27,28}$ del Departamento de Investigación sobre Tabaco del Instituto Nacional de Salud Pública (INSP), realizaron un estudio piloto, entre octubre de 2001 a mayo de 2002, en la Delegación Morelos del Instituto Mexicano del Seguro Social (IMSS), utilizando la metodología de consenso de Guías Diagnóstico Terapéuticas y "Casos Tipo" por enfermedad. Los costos anuales (estimados en pesos mexicanos de 2001) de la atención médica de los casos en su etapa diagnóstica y de primer año de tratamiento correspondieron a $\$ 58982$ para el caso de infarto agudo del miocardio (IAM) leve, a $\$ 84668$ para el caso IAM severo; para EPOC estable, \$6 668 y para el caso exacerbado, \$139 978; para el cáncer de pulmón (CP) en estadio IIB, \$54 473 y para el caso en estadio IV, \$107 520. Los costos anuales de atención médica por enfermedad ascendieron a \$27 199583 por IAM, a \$114 645892 por EPOC y a \$10 323745 por $\mathrm{CP}$, de los cuales son atribuibles al consumo de tabaco $\$ 123748$ 848, que corresponde a 7.3\% del presupuesto anual de la Delegación (cuadro IV).

Estos costos se encuentran subestimados ya que no contemplan la totalidad de enfermedades relacionadas con el consumo de tabaco, ni los costos por pérdidas de productividad, los cuales, según estudios internacionales, podrían llegar a ser tres veces más que los costos de atención médica. Dado que este estudio se realizó desde la perspectiva del proveedor, teniendo en cuenta la atención médica en el I y II nivel de atención durante el

Cuadro III

Estimaciones anuales de los costos en salud atribuibles al consumo de tabaco. América Latina

\begin{tabular}{lccccc} 
Estudio & $\begin{array}{c}\text { Año } \\
\text { estimación }\end{array}$ & $\begin{array}{c}\text { Servicios } \\
\text { incluidos* }\end{array}$ & $\begin{array}{c}\text { Enfermedades } \\
\text { incluidas }{ }^{\ddagger}\end{array}$ & $\begin{array}{c}\text { Costos médicos } \\
\text { atribuibles al tabaco }\end{array}$ & $\%$ PIB \\
Puerto Rico & 1983 & F H L M O P & C G M R O* & US Million & 0.43 \\
\hline Dietz et al (I99I) & 1997 & $\mathrm{H}^{\#}$ & C G R O & Million Bolívares & 0.3 \\
Venezuela & & & & 129 &
\end{tabular}

\footnotetext{
* Servicios: F: Pagos a otros profesionales; H:Atención hospitalaria; L: Cuidados a largo plazo; M: Medicina; O:Visitas ambulatoria;. P: Pagos a médicos

\# Enfermedades: C: Cardiovasculares; G: Enfermedades gastrointestinales; Is: Enfermedades isquémicas; M: Enfermedades malignas; R: Enf. respiratorias no maligna;. F: Costos ocasionados por incendios; O: Otros

$\S$ Otras enfermedades como tuberculosis y condicioines pediátricas

\# Incluye habitación básica hospitalaria, omite pruebas, cuidados intensivos y otros servicios

Fuente: referencia 13.
} 
año posterior al diagnóstico del caso de enfermedad, los resultados son válidos aunque extrapolables únicamente a la población del IMSS de Morelos.

A pesar de tener suficiente evidencia científica que sustenta la teoría de que el tabaquismo incrementa los costos de atención médica, existe una gran variabilidad en las estimaciones obtenidas (cuadros I-IV), lo cual no sólo ha generado un debate de tipo metodológico, sino que ha llevado al cuestionamiento de los hallazgos y resultados. Las estimaciones que han utilizado una metodología con enfoque transversal muestran una marcada diferencia de costos de atención médica cuando se comparan los fumadores, ex fumadores y no fumadores; sin embargo, cuando las comparaciones se realizan en forma longitudinal la diferencia en los costos entre los fumadores y no fumadores disminuye, e incluso en algunos estudios llega a ser menor entre los fumadores, comparado con los no fumadores.

\section{Estudios Iongitudinales}

Los estudios de costos de atención médica con perspectiva longitudinal son aquellos que basan sus estimaciones teniendo en cuenta los costos generados durante el tiempo de vida de un fumador, comparado con los generados en el mismo tiempo por un no fumador.

El cuadro V resume los estudios realizados con perspectiva longitudinal y los detalles metodológicos utilizados en cada uno ellos.
Leu y Schaub ${ }^{29}$ condujeron el primer estudio con enfoque longitudinal a principios de los años 80 en Suiza y encontraron que si bien era cierto que había un incremento en la utilización de los servicios hospitalarios y ambulatorios por parte de los fumadores, la corta esperanza de vida de éstos compensaba los costos de atención médica con el incremento en la utilización. La metodología de atribución de costos está basada en las tasas de mortalidad y el análisis fue limitado a los costos de atención médica. Un segundo estudio que afinó la metodología mediante un modelo econométrico demostró que los fumadores utilizan poco los servicios médicos ambulatorios pero tienen mayor número de días de hospitalización. Con estos dos resultados los autores concluyen que el tabaquismo no incrementa los costos de atención médica a lo largo de la vida.

Oster y colaboradore ${ }^{30}$ estudiaron las tres enfermedades más relevantes asociadas con el consumo de tabaco utilizando la incidencia de la enfermedad y sus costos; calcularon los costos directos e indirectos de los fumadores teniendo en cuenta la edad, el género y la intensidad del consumo de tabaco.

Manning y colaboradores ${ }^{31}$, desde una perspectiva social, utilizaron información procedente de las encuestas nacionales de salud, las aseguradoras de vida, riesgos y salud, además del sistema nacional de justicia. Los resultados del estudio refieren que los no fumadores subsidian a los fumadores en términos de atención médica y seguros de vida, pero en con-

\section{Costos de atención médica atribuibles al consumo de tabaco. IMSS Morelos. 200 I}

\begin{tabular}{|c|c|c|c|c|}
\hline Enfermedad & $\begin{array}{c}\text { Costo promedio } \\
\text { anual }\end{array}$ & $\begin{array}{c}\text { Casos } \\
\text { incidentes }\end{array}$ & $\begin{array}{l}\text { Casos atribuibles } \\
\text { al consumo de tabaco }\end{array}$ & $\begin{array}{c}\text { Costos médicos atribuibles } \\
\text { al tabaco* }\end{array}$ \\
\hline Infarto agudo del miocardio & 79531 & 342 & $192(154-226)$ & 15231739 \\
\hline FAP:045 $(0.35-0.55)^{\S}$ & & & & (I2 $239790-1795$ I 692) \\
\hline Enfermedad pulmonar obstructiva crónica & 73303 & 1564 & $136 \mid(|267-| 423)$ & $9974 \mid 926$ \\
\hline FAP:080 $(0.70-0.90)^{\#}$ & & & & $(92863 \quad 173-104327762)$ \\
\hline Cáncer de pulmón & 102215 & 101 & $86(73-91)$ & 8775183 \\
\hline FAP:085 $(0.75-0.95)^{\#}$ & & & & $(7433$ 096-9 29| 370) \\
\hline
\end{tabular}

\footnotetext{
* Valuado en pesos mexicanos de $200 \mathrm{I}$, teniendo en cuenta la metodología de consenso de panel de expertos

‡ Costo promedio anual ponderado por la proporción de casos según grado de severidad de la enfermedad

$\S$ FAP para IAM estimada en población derechohabiente de 35 a 65 años del IMSS de Morelos. Salazar et al

\# FAP para EPOC y CP estimada por el Centro para el Control de Enfermedades de EUA (CDC)
}

Fuente: Referencia 28 


\section{Cuadro $v$ \\ Estimaciones de tiempo de VIDA de los costos en SALUd ATRIBUibles AL CONSUMO DE TABACO}

\begin{tabular}{|c|c|c|c|}
\hline & & & (Dólares)* (\$USD) \\
\hline $\begin{array}{l}\text { Autor } \\
\text { año publicación }\end{array}$ & $\begin{array}{l}\text { Año } \\
\text { estimación }\end{array}$ & Lugar & $\begin{array}{lccc}\text { Costos } & \text { Costos de } & \text { Costos } & \text { Costos } \\
\text { totales } & \text { atención médica } & \text { morbilidad } & \text { mortalidad }\end{array}$ \\
\hline Oster et al $(1984)^{30}$ & 1980 & EUA & $\begin{array}{l}\text { Costo total } \$ 1000 \text { - } 61000 \text { dependiendo } \\
\text { del número de paquetes de cigarrillos fumados. } \\
\text { Los beneficios por dejar de fumar: } \$ 400-41,000 \\
\text { dependiendo del número de paquetes fumados }\end{array}$ \\
\hline Manning et al (1989) 31 & 1986 & EUA & $\$ 0.26$ por paquete \\
\hline Lippiat $(1990)^{32}$ & 1986 & EUA & $\begin{array}{l}\text { Costo incremental } \$ 281 \text { por año de vida } \\
\text { adicional de los no fumadores }\end{array}$ \\
\hline Hodgson $(1992)^{33}$ & 1990 & EUA & $\begin{array}{l}\$ 187 \text { billones durante los primeros cinco años a } \\
\text { partir de la linea de base }\end{array}$ \\
\hline Viscussi $(1995)^{34}$ & 1993 & EUA & $\begin{array}{l}\text { Reducción de los costos comparado con los } \\
\text { no fumadores, principalmente debido a ahorros } \\
\text { en pensiones y la seguridad social }\end{array}$ \\
\hline
\end{tabular}

Fuente: Max W. The financial impact of smoking on health-related cost: A review of the literature. Am J Health Promot 200I;I5(5):32I-33I

traposición los fumadores subsidian a los no fumadores en el ámbito de las pensiones y los cuidados de enfermería en casa. Estos resultados concluyen que los fumadores pagan su vicio con los niveles de impuestos de los cigarrillos; sin embargo, como política de salud pública habría que realizar un alza considerable en los impuestos para reducir el número de adolescentes fumadores.

Lippiat $^{32}$ retomó el estudio de Oster, lo reanalizó, y encontró que los datos se encuentran subestimados, ya que no se tienen en cuenta los costos por pérdida de productividad y muerte prematura.

Hodgson $^{33}$ incorporó un nuevo concepto en la metodología introduciendo un análisis de ciclo de vida, tomando como referencia un ciclo de 5 años a partir de la línea de base. Concluyó que en ese período el efecto acumulado del exceso en el cuidado médico era mayor en todos los grupos de edad, a pesar de su corta expectativa de vida, y que, además, los fumadores incurren en gastos más altos durante su vida, comparado con los no fumadores.

Viscussi, ${ }^{34}$ basado en el supuesto de que los fumadores comprenden perfectamente las consecuencias de su comportamiento frente al riesgo de enfermedad (morbilidad) y muerte (mortalidad) secundaria al consumo de tabaco, encontró que los fumadores ahorran dinero a la sociedad. Los ahorros son secundarios a una menor utilización de cuidados de enfermería y reducción del pago de pensiones.
Sloan y colaboradores ${ }^{35}$ realizaron un estudio longitudinal de los costos privados y sociales del tabaquismo. Los costos del tabaquismo de un hombre fumador de 24 años de edad son de USD \$183,000, y de una mujer fumadora de la misma edad, de USD $\$ 86,000$, durante la vida. Si se incluyen los costos privados y los impuestos a terceros (por exposición a humo de tabaco de segunda mano y pago a la seguridad social), los costos ascienden a USD $\$ 220,000$ en los hombres y 106,000 para las mujeres. El costo por paquete de cigarrillo durante toda la vida del fumador apenas alcanza los USD $\$ 40$.

\section{Discusión}

En esta revisión de la literatura publicada en los países de América, podemos observar que a través del tiempo los estudios de costos de atención médica atribuibles al consumo de tabaco han mejorado sustancialmente la selección de las fuentes de información, el tipo de análisis econométrico y, específicamente, la metodología de la atribución de los costos de atención médica al consumo de tabaco.

La metodología de la atribución de los costos al consumo de tabaco se basa en el concepto epidemiológico de causa-efecto, donde un factor de riesgo (consumo de tabaco) se asocia causalmente a la ocurrencia de un evento (enfermedad o muerte). Tal asociación causal debe de cumplir con los criterios de causalidad 
de Bradford Hill.* Este concepto epidemiológico ha sido extrapolado al concepto económico para efectos de atribución de costos al consumo de tabaco; por lo tanto, la estimación de la medida de asociación (riesgo) debe ser válida y consistente y, además, extrapolable a la población de estudio.

Inicialmente, la asociación causal se establecía comparando simplemente el riesgo de desarrollar el evento (enfermedad o muerte) entre los fumadores y los no fumadores, y esta medición se encontraba subestimada porque no reflejaba la magnitud de la exposición ni la historia de consumo de tabaco. En la actualidad esta asociación debe tener en cuenta al menos tres categorías fundamentales: fumador, ex fumador y nunca fumador. Esto permite comparar las dos categorías de riesgo (fumador y ex fumador) con la de nunca fumador, mejorando así el estimador.

Posteriormente se han afinado las estimaciones teniendo en cuenta el número de paquetes consumidos en la vida y el número de cigarrillos consumidos al día, además de ajustar las estimaciones por otros factores de riesgo como edad, género y características de estilo de vida. Esto hace que tanto las estimaciones de riesgo como las de los costos de atención médica sean más precisas y consistentes. Por ejemplo, en el estudio de Oster y colaboradores, ${ }^{30}$ se concluye que los costos económicos del tabaquismo varían considerablemente de acuerdo con la edad, el género y la cantidad de cigarrillos fumados. Los costos para un hombre de 40 años de edad que fuma sólo una caja de cigarrillos/día son de USD\$20 000, mientras para uno que fuma dos cajas de cigarrillos/día ascienden a USD \$56 000.

Si se tiene en cuenta la perspectiva anual, el tabaquismo incrementa los costos de atención médica y resulta en pérdidas de tiempo laboral, con efectos en la carga financiera de los fumadores, sus familiares, aseguradores y empleadores. Esto es evidente en todos los estudios realizados tanto en países desarrollados como en desarrollo.

Al tener en cuenta la perspectiva longitudinal, el tabaquismo incrementa los costos de atención médica como lo demuestra el estudio de Lippiat, donde simula que una disminución de $1 \%$ en las ventas de cigarrillos en los EUA incrementa la esperanza de vida en 1.45 millones de años, lo que se refleja en un aumento de los costos de atención médica en USD \$405 millones. ${ }^{32}$

\footnotetext{
* Criterios de causalidad de Bradford Hill: una asociación causaefecto entre un factor de riesgo y un evento debe cumplir con los siguientes criterios: a) temporalidad, b) fuerza de asociación, c) magnitud, d) dosis-respuesta, e) consistencia y f) plausibilidad biológica.
}

Los costos indirectos siempre deben ser considerados ya que son sustanciales. Estimaciones de estos costos muestran que pueden llegar a ser hasta tres cuartas partes o 40 a $60 \%$ de los costos totales atribuibles al consumo de tabaco. Se ha estimado que en los países desarrollados las pérdidas de productividad corresponden aproximadamente a tres veces más los costos médicos directos. ${ }^{6,13}$

Barendregt y colaboradores ${ }^{36}$ enfocan su estudio en tres enfermedades, demostrando que los ahorros en los costos de atención médica se logran a corto plazo si y sólo si todos los fumadores dejaran de fumar. En el análisis a largo plazo los costos de atención médica se incrementan. A pesar de que el autor no consideró los costos de atención de los ex fumadores, es importante tener presente que a menudo los fumadores dejan de fumar cuando ya han presentado la enfermedad.

\section{Conclusiones}

$\mathrm{Al}$ consumo de tabaco no se le reconoce ningún aporte directo o indirecto compatible con la salud poblacional. Quien consuma una cajetilla diaria, irremediablemente acabará enfermando y eventualmente morirá en forma prematura. El costo del tratamiento médico de cualquier enfermedad relacionada con el consumo de tabaco durante un año cualquiera supera exponencialmente cualquier contribución hecha por un comprador de este producto en el mismo periodo.

Para diseñar políticas de control del tabaquismo es preciso comprender el comportamiento de la epidemia en nuestros países y, además, se requieren esfuerzos coordinados de un grupo interdisciplinario, donde las medidas de control médico y de salud pública vayan de la mano de las medidas económicas.

En el ámbito clínico, se requiere capacitar a los profesionales de la salud, no sólo para mejorar el sistema de registro de la epidemia de tabaquismo sino para brindar una consejería individualizada al fumador. Esto implica una inversión en el desarrollo del capital humano que trabaja en salud, comenzando por el cambio de perspectiva del concepto de salud -enfermedad en las facultades de las ciencias de la salud y la reestructuración del curriculo académico.

En la estructura y organización del sistema de salud se requiere una fuerte inversión en programas estatales y locales orientados a prevenir y desincentivar el consumo de tabaco, mediante la creación de programas escolares y comunitarios, así como el fortalecimiento de las clínicas de cesación de consumo de tabaco.

Para desarrollar medidas de control de tipo económico, específicamente la fiscal, se requiere de evidencia científica documentada de los costos de atención médica en el país, considerando los costos directos, los 
indirectos y las estimaciones de los efectos en la salud de los no fumadores por exposición a humo de segunda mano en el hogar y en los lugares de trabajo (costos por externalidades).

La experiencia ha demostrado que existen medidas de control del tabaco costo-efectivas que pueden ser utilizadas en diferentes ámbitos: en el poblacional, el incremento de los precios y de los impuestos al tabaco, la prohibición total de la publicidad directa e indirecta, la instauración de todos los lugares públicos libres de humo de tabaco y la colocación de los mensajes gráficos en las cajetillas; en el económico, la medida más costo-efectiva para reducir el consumo de tabaco es la elevación de los precios por vía de los impuestos. Esta medida, además de contribuir positivamente en la recaudación fiscal, es la que a largo plazo reducirá el mayor número de muertes y enfermedades atribuibles al consumo de tabaco y con ello aliviará la carga económica y social que esto representa para los gobiernos y los sistemas de salud. ${ }^{18}$

En los países latinoamericanos se requiere de un esfuerzo conjunto para establecer un sistema de vigilancia epidemiológica que incluya información epidemiológica, económica, los determinantes socioeconómicos, las políticas de control y las actividades de la industria tabacalera. Se ha demostrado que un buen sistema de vigilancia epidemiológica permite el monitoreo de la epidemia y la evaluación del impacto de las políticas de control.

El fortalecimiento de las capacidades técnicas y del recurso humano que trabaja en salud pública es otro de los retos de aquellos que lideran hoy el control del tabaco en América; sólo así se tendrán datos válidos y oportunos para la toma de decisiones.

\section{Referencias}

I.World Bank. Curbing the epidemic: Governments and economics of tobacco control. Washington, DC: World Bank; 1999.

2. Departamento de Salud y Servicios Sociales de los Estados Unidos de América. Tabaquismo y Salud en las Américas. Publicación No. (CDC) 92-8420. Atlanta, Georgia: USDHHS, 1992:9-I5.

3. Kumra V, Markoff BA.Who's smoking now? The epidemiology of tobacco use in the United States and abroad. Clin Chest Med 2000:21; I-9.

4. Warner K, Hodgson TA, Carroll C. Medical cost of smoking in the United States: Estimates, their validity, and their implications. Tob control 1999; 8:290-300.

5. Leistikow BN. The human and financial costs of smoking. En: Smoking and pulmonary and cardiovascular diseases. Clin Chest Med 2000;2I(I):189-195.

6. Max W. The financial impact of smoking on health-related cost:A review of the literature. Am J Health Promot 200I;15(5):32I-33I.
7. U.S. Department of Health, Education and Welfare. Smoking and Health. Report of the Advisory Committee to the Surgeon General of the Public Health Service. Publication No. (PHS) I I03. Washington, DC: USDHEW, 1964:33-42.

8. U.S. Department of Health and Human Services. The Health Consequences of Smoking: Cancer.A Report of the Surgeon General. Publication No. (PHS) 82-50 179. Washington, DC: USDHHS, I982: I-II. 9. U.S. Department of Health and Human Services (USDHHS). The Health Consequences of Smoking: Cardiovascular Disease. A Report of the Surgeon General. Publication No. (PHS) 84-50204. Rockville (MD): USDHHS, I983:I-II.

10. Great Britain Scientific Committee on Tobacco and Health Great Britain Department of Health Scientific Committee on Tobacco and Health. Report of the Scientific Committee on Tobacco and Health. London: Pitman Medical Publishing Co., 1988:3-8.

II. Samet J. Los riesgos del tabaquismo activo y pasivo. Salud Publica Mex 2002;44 (SI):SI44-SI60.

12. U S Department of Health and Human Services (USDHHS). The health consequences of smoking:A Report of the Surgeon General. Atlanta (GA): U S Department of Health and Human Services. Centers for Disease Control and Prevention. National Center for Chronic Disease Prevention and Health Promotion, Office on Smoking and Health; 2004.

13. Lightwood J, Collins D, Lapsley H, Novotny T. Estimating the costs of tobacco use. En: Jha P, Chaloupka F, ed. Tobacco control in developing countries. Oxford: Oxford University Press, 2000: 63-99.

14. Hendrick JL. The economic cost of cigarette smoking. HSMHA Health Rep 1971;86:179-182.

15. Kristein MM. Economic issues in prevention. Prev Med 1977;6:252-264. 16. Luce BR, Schweitzer SO. Smoking and alcohol abuse:A comparison of their economic consequences. N Engl J Med 1978;298:569-57I. 17. Office of Technology Assessment (OTA), U S Congress. Smokingrelated deaths and financial costs. Washington, DC: Health Program, OTA; 1985. OTA staff memorandum.

18. Rice DP, Hodgson TA, Sinsheimer P,Browner W, Kopstein AN. The economic costs of the health effects of smoking, 1984. Milbank $Q$ 1986;64 (4):489-547.

19. Barlett JC, Miller LS, Rice DP, Max W. Medical expenditures attributable to cigarrette smoking -United States, 1993. MMWR Morb Mortal Wkly Rep 1994;43:469-472.

20. Miller LS, Zhang X, Rice DP, Max W. State estimates of total medical expenditures attributable to cigarette smoking, 1993. Public Health Rep | 998; | | 3:447-458.

21. Miller VP, Ernst C, Collin F. Smoking-attributable medical care costs in the USA. Soc Sci Med 1999;48:375-391.

22. Warner K. The economics of tobacco: Myths and realities. Tob Control 2000;9:78-89.

23. Zeger SL, Wyant T, Miller LS, Samet J. Statistical testimony on damages in Minnesota v. Tobacco industry. En: Gastwirth J. Statistical in the Courtroom. Nueva York (NY): Springer-Verlag; 2000.

24. Johnson E, Dominici F, Griswold M, Zeger SL. Disease cases and their medical costs attributable to smoking: An analysis of the national medical expenditures survey.J Econometrics 2003;||2:|35-|5|. 25. Dominici F, Zeger SL. Smooth quantile ratio estimation with regression: Estimating medical expenditures for smoking attributable diseases. Biostatistics 2005;6:505-5 I9.

26. Arredondo A, Parada I, Carrillo C. Financial consequences of changes in health care demands related to tobacco consumption in Mexico: Information for policy makers. Health Policy 2002;61:43-45.

27. Reynales-Shigematsu LM, Juárez-Márquez SA, Hernández-Ávila M. Costos de la atención médica de enfermedades atribuibles al consumo de tabaco. En: Primer informe sobre combate al tabaquismo. México ante el Convenio Marco para el Control del Tabaco. México. Cuernavaca: Instituto Nacional de Salud Pública; 2005:77-85. 
28. Reynales-Shigematsu LM, Juárez-Márquez SA,Valdés-Salgado R. Costos de atención médica atribuibles al tabaquismo en el IMSS, Morelos. Salud Publica Mex 2005;47(6):45I-457.

29. Leu Re, Schaub T. Does smoking increase medical care expenditure? Soc Sci Med 1983;17(23):1907-1914.

30. Oster G, Colditz GA, Kelly NL. The economic cost of smoking and benefits of quitting for individual smokers. Prev Med 1984;13:377-389. 31. Manning WG, Keeler EB, Newhouse JP, Sloss EM, Waserman J. The taxes of sin. Do smokers and drinkers pay their way? JAMA 1989;26|:1604-1609.

32. Lippiat BC. Measuring medical cost and life expectancy impacts of changes in cigarette sales. Prev Med 1990;19:5।5-532.
33. Hodgson T. Cigarette Smoking and lifetime Medical Expenditures. Milbank Q 1992;70(I):81-125.

34.Viscusi WK. Cigarette taxation and the social consequences of smoking. En Tax policy and the economy: Cambridge: National Bureau of economic research/ MIT Press, 1995.

35. Sloan F, Ostermann J, Picone G, Conover C, Taylor Jr. D. The price of smoking. Cambridge:The MIT Press; 2004.

36. Barendregt J], Bonneu L,Van Der Mass PJ. The health care costs of smoking. N Engl J Med 1997;337:1052-1057. 